\title{
Person-centred, integrated and pro-active care for multi-morbid elderly with advanced care needs: a propensity score- matched controlled trial
}

\author{
G. K. R. Berntsen ${ }^{1,5^{*}}$, M. Dalbakk², J. S. Hurley ${ }^{1}$, T. Bergmo ${ }^{1}$, B. Solbakken², L. Spansvoll', J. G. Bellika' , S. O. Skrøvseth',
}

T. Brattland ${ }^{4}$ and M. Rumpsfeld ${ }^{2}$

\begin{abstract}
Background: Person-centred care (PCC) focusing on personalised goals and care plans derived from "What matters to you?" has an impact on single disease outcomes, but studies on multi-morbid elderly are lacking. Furthermore, the combination of PCC, Integrated Care (IC) and Pro-active care are widely recognised as desirable for multimorbid elderly, yet previous studies focus on single components only, leaving synergies unexplored. The effect of a synergistic intervention, which implements 1) Person-centred goal-oriented care driven by "What matters to you?" with 2) IC and 3) pro-active care is unknown.

Methods: Inspired by theoretical foundations, complexity science, previous health service research and a patientdriven evaluation of care quality, we designed the Patient-Centred Team (PACT) intervention across primary and secondary care. The PACT team collaborate with the patient to make and deliver a person-centred, integrated and proactive multi-morbidity care-plan. The control group receives conventional care. The study design is a pragmatic six months prospective, controlled clinical trial based on hospital electronic health record data of 439 multi-morbid frail elderly at risk for emergency (re) admissions referred to PACT and 779 propensity score matched controls in Norway, 2014-2016. Outcomes are emergency admissions, the sum of emergency inpatient bed days, 30-day readmissions, planned and emergency outpatient visits and mortality at three and six months follow-up.
\end{abstract}

Results: The Rate Ratios (RR) for emergency admissions was 0,9 (95\% Cl: 0,82-0,99), for sum of emergency bed days 0,68 (95\%Cl:0,52-0,79) and for 30-days emergency readmissions 0,72 (95\%Cl: 0,41-1,24). RRs were 2,3 (95\%Cl: 2,02-2, $55)$ and $0,9(95 \% \mathrm{Cl}: 0,68-1,20)$ for planned and emergency outpatient visits respectively. The RR for death at 3 months was 0,39 (95\% Cl: 0,22-0,70) and 0,57 (95\% Cl: 0,34-0,94) at 6 months.

Conclusion: Compared with propensity score matched controls, the care process of frail multi-morbid elderly who received the PACT intervention had a reduced risk of high-level emergency care, increased use of low-level planned care, and substantially reduced mortality risk. Further study of process differences between groups is warranted to understand the genesis of these results better.

Trial registration: ClinicalTrials.gov (identifier: NCT02541474), registered Sept 2015.

Keywords: Person-centred care, Integrated care, Proactive care, Health care utilisation, Mortality, Propensity score matched controls

\footnotetext{
* Correspondence: gro.rosvold.berntsen@ehealthresearch.no

${ }^{1}$ Norwegian Center for e-health research, University Hospital of North

Norway Trust, Universitetssykehuset, PB 35, 9038 Tromsø, Norway

${ }^{5}$ Institute of community medicine, UiT The Arctic University of Norway, PO

Box 6050 Langnes, N-9037 Tromsø, Norway

Full list of author information is available at the end of the article
}

(c) The Author(s). 2019 Open Access This article is distributed under the terms of the Creative Commons Attribution 4.0 International License (http://creativecommons.org/licenses/by/4.0/), which permits unrestricted use, distribution, and

reproduction in any medium, provided you give appropriate credit to the original author(s) and the source, provide a link to the Creative Commons license, and indicate if changes were made. The Creative Commons Public Domain Dedication waiver (http://creativecommons.org/publicdomain/zero/1.0/) applies to the data made available in this article, unless otherwise stated. 


\section{Background}

A rising number of elderly citizens heralds an increased number of persons with complex long-term needs (CLNs) who need and expect more of our care systems. Persons with CLNs typically face multiple care providers, organisations and specialists [1], and are especially vulnerable to care fragmentation [1-4]. This group also dominates the $5-10 \%$ top spenders who account for $2 / 3$ of high-level health care spending both in Norway and internationally [5-7]. The expected increase in persons with CLN is threatening the sustainability of our healthcare systems [8]. Current care systems, designed for acute short-term needs, are struggling to meet the rising tide of persons with CLNs.

An extensive body of research indicates that critical elements of high-quality care for persons with CLNs include strong primary care with an inherent whole person, integrated and pro-active focus [9-14]. For simplicity, we will nickname this three-part solution PIP care, for Person-centred care (PCC), Integrated Care (IC) and Proactive care (Pro-C). There is an abundance of generally promising reviews regarding each of these three elements [15-24]. Yet, outcome evaluations remain unpredictable or weak with a high proportion of negative studies [15-22]. Reviewers report that there is little conceptual clarity on how to operationalise the PIP-elements, and for whom and in which contexts they will work $[16,17,25]$. We propose that the PACT study differs from previous research in two regards:

\section{A synergistic approach}

Even though international recommendations highlight the complex and multi-faceted synergies between these three PIP-elements, studies in this field have generally followed the traditional approaches of the "rational-linear" approach, "... When designing intervention and implementation strategies, as well as when conducting rigorous evaluations, there is a tendency to reduce messy real world situations into the individual component parts in an attempt to determine the relationships between them" [20, 26]. However, to achieve better outcomes for persons with CLNs, the whole chain of care needs to change. We propose that the PIP-components are interdependent, and will only produce the expected effects if all the components are in place. The effect of an intervention where the PIP elements work synergistically together is mostly unexplored.

\section{A sharper definition of PCC}

PCC is in many ways the foundation of PIP-care. PCC is widely recognised as desirable $[13,15,27]$ yet the gap between the rhetoric and reality of PCC remains uncomfortably wide $[28,29]$. The last few years, a sharper vision of PCC described by Bisognano as "flipped healthcare" is emerging [15, 30, 31]. It flips care focus from: What is the matter?" to "What matters to you?" It forces attention from professionally defined diagnosis and functional goals to personal goals. Here, we prefer the term "Goal-oriented Person-centred care" (Goal-PCC), to underline that in "flipped care," the entire chain of care, including care planning, delivery, and evaluation is co-created with the person and driven by personal goals [32]. While Coulter found in her Cochrane review that Goal-PCC had a small but significant impact on outcomes in single disease contexts, neither Coulter nor we have found any studies that applied this concept to multi-morbid patients [15].

Norway has a robust primary care system [33], with high accessibility and low out of pocket expenses, among the highest life expectancies in the world, and excellent health outcomes in international comparisons, and a commitment to quality improvement for persons with CLNs [34-39]. However, persons with CLNs in Norway report the same challenges as other high-income countries, making the Norwegian context internationally relevant [39-41].

We designed the PACT intervention to address quality challenges identified by patients with CLNs in Norway [41], in alignment with the theoretical and empirical foundations of chronic care ideals. Central ideals were the Chronic Care Model [42], Goal-oriented Person-centred care [15, 31, 41, 43, 44], Shared Decision Making [45], integrated care pathways [46] and pro-active risk management $[23,47]$. We were mindful of the complex nature of both the health service and the PACT intervention [48-50]. The care process for each person, here denoted as the individualised Patient Pathway (iPP) [41], is a product of a complex adaptive system (CAS) [49]. Key elements to support problem-solving in a CAS are: A good enough vision or goal, simple guiding rules and a wide space for innovation and creativity to reach common goals [49]. The goals and rules were set out in our previous work on the Person-centred integrated care quality framework [41]. Briefly, the iPP serves patient-defined goals [31, 44], with attention to PCC, IC and Pro-C, while incorporating insights and feedback from both the patient and other contributors is an ongoing attribute of the care process [48]. To the best of our knowledge, we have not found other studies on the effects of a complex, synergistic and "flipped" Goal-PCC model, which builds all three PIPelements into the care model the way our Patient-Centred Team (PACT) intervention does.

\section{Aim}

The PACT study aims to improve the triple aim of patient care experience, health outcomes and cost-benefit ratios for persons with CLNs. Due to delays in the collection of patient reported outcomes (see details later), we here report on early and available health outcomes 
using routine data from the electronic health record (EHR). We expect that the PACT intervention applied to a population of high risk frail multi-morbid elderly will stabilize the patient's health situation through improved quality of care. As a result, we expect to see a reduced use of high-level emergency care and a higher use of low-level planned care. Hypothetically, use of emergency care could be reduced simply by denying patients adequate care. This would of course be unethical, and in this frail group, we would expect increased mortality. We therefore needed to show that reductions in the use of high-level emergency care was not accompanied by increased mortality. To summarize, the hypotheses $(\mathrm{H})$ examined in this paper are that the PACT intervention will cause:

- H1: Reduced use of high-level emergency care .

- H2: Increased use of low-level planned care

- H3: Unchanged mortality risk

\section{Methods}

\section{Study design, reporting and protocol changes}

This study is a prospective propensity score (PS) matched controlled trial evaluating the effectiveness of the PACT service. Briefly, the use of routine data from the EHR, and the propensity score techniques described by Rubin [51-53], allowed us to create a valid comparison group even when the target population was defined by a discretionary referral process. The same EHR data also contained relevant process and outcome measures, which enabled a comparison of intervention and usual care. We follow recommendations for evaluation of complex interventions [54], complexity science [55] and quality improvement methodology [56].

The study design, which has been called a synthetic RCT, lies somewhere between the randomized controlled trial (RCT) and the observational study. We adhere to the CONSORT [57] STROBE [58], TIDieR [59] and the SAMPL [60] checklists for reporting on RCT, observational, intervention and biostatistical studies respectively.

We have previously published our study protocol at ClinicalTrials.gov (identifier: NCT02541474) and as a protocol paper [61]. We found it necessary to make the following protocol changes: 1) Due to delays in data collection, we report on secondary health outcomes before we are able to report on the primary outcome: the patient reported health experience by the SF12 questionnaire [62]. 2) Due to a high risk of healthy selection bias, we were allowed to include routine EHR data on all patients receiving PACT without their consent, while the original protocol planned inclusion based on written informed consent.

\section{The arguments for these two changes are}

Patients, who had consented to receive care (care consent) from the PACT team, were eligible to be invited to give their informed written consent to participate in the PACT study (study consent). However, a high proportion suffered from acute serious or overwhelming morbidity at baseline. It is unethical to ask patients to fill a study consent or questionnaire in a situation where treatment and adjustment should take precedence. When patients had recovered sufficiently for study recruitment to be ethical, many were already past the 4 week study recruitment window and were no longer eligible. 2) A high proportion of persons with cognitive impairment were unable to provide informed consent and we found that family members were frequently uncomfortable answering personal questions on the patient's behalf. For these two reasons, the recruitment and collection of the primary outcome based on patient reported data is delayed, is still ongoing and will continue to March 2019.

As early results on secondary health outcomes were important for health service decision makers regarding the funding of the PACT service, we looked for other ways to evaluate the PACT service. We also feared that a study based on informed consent would be flawed by a healthy selection bias. With this background, we sought and were granted permission (see ethics section) to extract routinely collected data from the EHR which contains both exposure and outcomes variables for both the intervention and control population, without asking for patient consent. This would ensure fair recruitment, irrespective of challenges to provide consent. The main ethical concern of this approach was to maintain privacy of the routine data provided to the research team. The privacy protection officer approved the project with the following precautions to ensure the privacy of the participants: We prepared pseudonymised analysis files, which means that direct identifiers such as names, addresses or id-numbers, were replaced with a pseudonym. We saved and used analysis files exclusively on "offline" devices/ computers. Only 3 researchers (GB, JH, TB1) had access to the analysis files. All intermediate and final analysis results are fully anonymous.

We, are therefore able to report here on the outcomes available to us from the EHR: healthcare utilisation and risk of death.

\section{The intervention design}

The intervention was tailored to each patient, as long as the PACT team adhered to the following structures and principles.

\section{The PACT structures}

The PACT team is a cross-organisational (hospital and municipality) multi-disciplinary geriatric team set in two 
separate municipalities (M1 and M2), both with more than 20,000 inhabitants in Norway. Both M1 and M2 provide typical primary care services, and both host a local hospital. The local PACT team includes nurse coordinators, physio- and occupational therapists, geriatric nurses, pharmacists, medical secretaries and a medical doctor. The hospital and municipalities share financial, management and employment responsibilities for the team members. The PACT board represented significant stakeholders: Patients, primary and secondary care, research and health technology.

\section{PACT care process}

We designed PACT in a heuristic iterative process cycling between literature reviews [31], listening to and addressing patient [41], professional and manager experiences. PACT has many of the same components as other interventions directed at frail elderly persons: PACT is person-centred, applies a comprehensive geriatric assessment methodology to create an evidence-based care plan. PACT applies a combination of a case manager and a multi-professional team to address complex patient issues in line with international guidelines [11, 12]. However, PACT differs from the bulk of integrated care research in two critical respects:

1. The vision of a Goal-oriented PCC: The heart of the PACT intervention is a continuous process of trust-building, sensitive exploration and cocreation between professional and patient, aimed at capturing "What matters to you?". Together with the person, PACT translates "What matters" into relevant goals for care and makes sure that all involved parties are aware of personal goals, and that each person's role relative to goals, is clear. Theoretically, this aligns with quality improvement projects and complexity science where goals/ vision define subsequent plans, process and evaluation $[49,56]$.

2. The synergetic cyclical care process: In the PACT intervention, the three PCC, IC and Pro-C elements synergistically build on each other. We hypothesise that Pro- $\mathrm{C}$ is most influential for health and functional outcomes, and that Pro-C relies on the presence of Goal-PCC and IC to achieve effects. The design of a care plan follows a cyclical pattern in line with quality improvement and complexity theory: The development of goals, care plan and delivery continuously adjust to new insights and feedback from the involved parties. The care process is described in more detail elsewhere (Additional file 1 - the PACT care process). Briefly, the
PACT team supports usual care in the creation of a goal-oriented, person-centred, integrated and pro-active care plan according to the following simple rules.

1) PCC, where "what matters" to the person drives decision making of the care plan.

2) IC includes comprehensive care planning by a multi-disciplinary, cross-organisational care team, designed to meet goals from 1 . The process involves the person, the PACT miniteam (see below) and the informal and formal long-term carers who will take over when PACT withdraws. It results in a negotiated care plan, which sets out the overarching personal goals (what matters), and then outlines roles and responsibilities for the long-term team: who does what, when, and why. PACT is mindful that a strong care plan ownership by both patient and the most involved health workers is essential for longterm success after PACT withdrawal.

3) Pro- $C$ includes risk management and preparation for early release to as low-level care as possible while ensuring that adequate evidence-based guidelines, such as comprehensive geriatric assessment [63], have been reviewed. If possible and desirable, PACT implements guidelines for the range of conditions present. Included in the team's repertoire are support for self-management, emergency contingency plans, diagnosis specific monitoring tools (i.e. signs of infection in COPD patients, weight increase in heart failure patients), and the "bow-tie" analysis [47]. To be included in the care plan, pro-active measures must align with personal goals from 1 and the advice of the multi-disciplinary team from 2 .

4) Continuous evaluation of both care planning and delivery, together with the person, to ensure dynamic learning and adjustment of the process in accordance with "what matters". The patient's feedback regarding the realisation of "what matters" is the guiding principle.

5) PACT starts the delivery of care while usual care resources are still being assembled/trained. PACT aspires to a gradual handoff of care delivery to self-management and usual care as soon as possible. PACT revisits the patient and his/ her care team for up to 3 months after withdrawal. If significant gaps in planned care occur during this time, PACT either temporarily fills the gap or re-assesses the plan to set it on course again. 
A core team consisting of a nurse coordinator, a physician and a secretary review all referrals. Patients admitted to the intervention are assigned a PACT mini-team consisting of a nurse, physician, physiotherapist, occupational therapist and pharmacist. One of these takes on the role as the primary coordinator of the patient pathway for the duration of PACT involvement. The mini-team have the freedom to "invent" the best possible care plan together with the person in question, as long as they are loyal to the above principles. Their work will consist of exploring patient goals, initiating diagnostic work-ups and other relevant evaluations, addressing the immediate clinical needs of the patient, and starting the coordination work regarding identifying and recruiting the usual care resources that need to be involved in long-term care planning and delivery when PACT withdraws. Handoff is thus a longitudinal collaborative process, where PACT works together with the patient and usual care to support them while a long-term care plan/ delivery comes into place. The average active PACT intervention time was 30 days.

PACT has a daily meeting involving all PACT employees, across the mini-teams, where the team members discuss both challenges at patient and system level. The PACT team project leader reports regularly on system level challenges to the PACT cross-organizational board.

\section{Data sources and quality}

The study extracted following variables for eligible populations in the four intervention and control municipalities from the EHR: Age, sex, start and stop time of outpatient, ambulatory care and inpatient episodes of care, degree of emergency for each episode, ICD-10 diagnoses and Diagnosis Related Group (DRG)-codes registered for each episode [64], DRG-points and date of death. Professionals collect these variables as a matter of routine care for all patients. The local hospital, which supplied our EHR-data, provides $97 \%$ or more of all out-patient and in-patient hospital services for the local population [65]. All Norwegian hospitals report these data routinely to the Norwegian Patient Registry (NPR). NPR consistently assesses data quality and corrects errors in the source data in collaboration with the source institution [65]. The EHR is updated with mortality data from the Norwegian Population Registry (PR) on a monthly basis, including all deaths (i.e. deaths at home, hospital or other institutions) in the study population. It takes approximately one week from when the physician issues a death certificate until the PR is updated (personal communication from $\mathrm{PR}$ ).

\section{Patients}

\section{Intervention patients - index episode}

The analysis includes all patients who consented to (care consent), and were offered treatment by the PACT team from Oct 2014 and Sept 2015 in M1 and M2 respectively until March 2016, with a 6month follow-up, ending in Sept 2016. As there is no agreed method of identification of patients with CLNs, PACT applied the following recruitment process. General practitioners, home-nursing services, hospital specialists or hospital nurses were encouraged to refer community-dwelling or hospitalised patients with multi-morbidity, complex longterm care needs and high short-term risk for emergency hospital (re-)admission based on their professional discretion (i.e., a clinical crisis).

- Inclusion criteria: Patients living in M1 or M2, aged $>60$ years, referred to a PACT-team to be reviewed for eligibility to receive PACT care and the patient provided an oral consent, recorded in the EHR, to receive PACT care (care consent).

- Exclusion criteria: The PACT team reviewed all referrals, and declined patients who had an adequate care plan, or were in no need of a multi-disciplinary follow-up. PACT declined these referrals within $<24$ h. They received no PACT services.

\section{Control patients - index episode}

All PACT referrals are indicative of a clinical crisis in a person with multi-morbidity. Patients $>60$ years, who experienced a clinical crisis as evidenced by an emergency admission (index episode), were eligible as controls. Controls were recruited from the pool of similar patients who had not received PACT care in the PACT intervention municipalities (M1, M2), and from two control municipalities (M3 and M4), where the PACT intervention was unavailable. M3 and M4 had a similar population size and local health care structure to M1 and M2 respectively.

Controls received usual care. Usual care consists of evidence based care for the cause of emergency admission to hospital. Depending on the patient's condition, care for other diagnoses is either achieved through referrals to the appropriate in-house specialist service, or through a recommendation for follow-up in the discharge letter directed at the GP. Care-coordination and integration at discharge is achieved through standard electronic communication and discharge routines designed in collaboration by the hospital and municipal care organizations.

\section{Analyses}

We followed intention to treat principle, as we included all patients who were offered and gave their consent to 
receive services from PACT services in analyses, independent of the actual length, content, and delivery of PACT services.

\section{Power, modelling decisions and statistical significance}

We made apriori power calculations for emergency admissions. These indicated a need for at least 390 patients in each group to show a $20 \%$ reduction in emergency admissions, ( $\beta$ of $80 \%$ and $\alpha$ of $0,05 \%$ ). We based power calculations on Norwegian hospital admissions data of patients with at least one admission last year (location parameter $\mu=0.5$ and scale parameter $\sigma=1.1$, non-parametric Wilcoxon two-sample test). [66]

To minimise type I error, we based analytic modelling decisions on the size of results rather than statistical significance, reserving statistical tests for evaluation of outcomes. We considered a change in point-estimates $>10 \%$ to signify a large enough confounding effect to warrant adjustment for the variable in the statistical model $[67,68]$. Similarly, we considered a $10 \%$ difference in effects between a main analysis and a sensitivity (sub-group) analysis to be large enough to warrant separate consideration in Results. We defined a probability of $<0,05$ as statistically significant. We report both exact $p$-values and $95 \%$ confidence intervals to reflect the statistical uncertainty of point estimates. The 95\% CI also conveys statistical significance at the chosen significance level, as a $95 \%$ confidence interval for a RR which includes 1 means that the null-hypothesis was not rejected. There were no interim analyses planned.

\section{Outcome measures}

We expected the PACT intervention to have its main effect through its Pro-C component, which prevents the need for emergency care. In the absence of increased mortality, we interpret less use of emergency resources as an indicator of better health and function. We hypothesised that increased use of planned care events at the lowest effective level of care would be instrumental in the reduction of high-level emergency care. Although death is the ultimate health outcome, we did not expect a substantial effect on mortality, as the literature did not support this [16]. We monitored deaths mainly to ensure that the mortality experience in the two groups was comparable and that the expected reductions in care utilisation was not attributable to increased mortality. Based on these expectations, we chose the following outcome measures within the observation period. Outcome measures by hypotheses were:

H1: We expect a reduced utilisation of high-level emergency care.

- Count of emergency admissions (defined as care required within $24 \mathrm{~h}$ ) - reflects the number of emergencies that could not be solved by municipal care.

- Sum of emergency in-patient bed days - is a composite measure reflecting both number of emergencies and length of stay resulting from each emergency.

- Count of re-admissions (emergency admissions within 30 days of last discharge) - is a measure of the quality of the discharge arrangements of the previous hospitalisation.

H2: Increased utilisation of low-level and planned care:

- Count of planned out-patient visits

- Count of emergency out-patient visits

H3: No change in mortality risk:

- Mortality risk at three and six months follow-up

The follow-up started at the time of the first referral to PACT (intervention) or at the time of emergency admission (controls) and ended at six months follow-up or death. Administrative personnel recorded outcomes as a matter of routine and were blind to group allocation.

\section{Matching, propensity score, and balance}

This is parallel arm study, with a 1:2 intervention-control ratio. We identified both a local (same municipality) and a distant control from a similar municipality without PACT services, for each intervention patient. In the pooled data, we worked with matched triplets: The intervention patient + their local + distant control.

Within the eligible control populations, we performed exact matching on sex, intervention-control site and year of the episode. Within these constraints, we identified controls with a propensity score of $+/-0,2$ SD calliper of the intervention patient's score using MatchIt software following the methodology laid out by Rubin and Rosenbaum [51, 52, $69,70]$. We used the following variables with a theoretical link to outcomes to build the propensity score: [71, 72] Demographic variables (age, sex), last year's care-utilisation variables, last two year's morbidity variables and risk scores reflecting resource utilisation, re-admission risk [73], and mortality.[77] Three per cent of intervention patients lacked hospital visits last year so we could not construct their propensity scores. Within callipers, we matched on Mahalanobis distance (MD) on the three variables that were most unbalanced after PS-matching, as recommended by Rubin [53]. We provide balance statistics in line with recommendations by Rubin [53].

\section{Regression analyses}

The purpose of regression analyses is to provide an as unbiased estimate of the main effect of treatment variable as possible. Even when there is an excellent balance, there may be residual confounding, which may require additional multivariate adjustment analyses of 
the matched dataset. In agreement with the data fit, we chose negative binomial regression for our count variables (sum bed days, admissions and outpatient visits) [75] and Cox regression for mortality risk. Using Kaplan Meier (KM) plots and proportional hazards (PH)-test, [76] we found the $\mathrm{PH}$ assumptions to be violated at 4-6 months, although the KM-lines did not cross (See Fig. 2). We report RRs for both 0-3 months and $0-6$ months. Readers should interpret the latter as an average RR for the full period. We chose robust estimations of standard errors in line with recommendations [75].

Exposure variables: We used both continuous and categorical variables as they were reported in the EHR, with the exception of Lead Days-variable (Number of days in hospital leading up to inclusion). Lead-days had a U-shaped relationship to several outcome variables, which is why we created a quintile nominal variable, and included both the nominal and continuous version in model building. None of the final main effect $95 \% \mathrm{CI}$ were unreasonably wide, so we did not consider collinearity a problem (See Table 3).

We built models according to a general rule set to minimise the effect of subjective modelling decisions on results. In a combined stepwise forwards / backwards selection process, we examined the confounding effects of variables with a statistically significant difference, or a standardised difference $>0,2$ SD between groups and the three compound variables on resource utilisation [64], re-admission risk [73], and mortality [74]. For each outcome, we added the corresponding "before-measure" to all models. For mortality, there are no before measures, so we included proxies for death-risk instead: age and the Elixhauser score [74]. Finally, we added the matched-triplet-ID as a random-effect variable to adjust for dependencies caused by matching [71]. The Higgins $\mathrm{I}^{2}$ statistic [77] showed heterogeneity across study sites, which is why we added "Site" both as a random and a fixed effect variable following Cochrane meta-analysis advice [78]. In sensitivity analyses, data from outlier sites were omitted to estimate their effect on results [77].

\section{Bias concerns}

Controls become eligible upon an emergency hospital admission while intervention patients become eligible upon referral to the PACT intervention. We outline the potential biases due to these differences, and how we have dealt with them in Table 1.

\section{Presentation of results and software}

To explore the bias concerns outlined above, we made the following sub-group analyses for all outcomes: 1) Survival and indication bias: Only controls who survived median lead-days of PACT patients, 2) Healthy selection bias: Only PACT patients who had an emergency admission index episode, and 3) strictest subgroup: a combination of 1 and 2 . In the

Table 1 Overview of bias concerns, consequences and adjustments in the PACT intervention study, Norway 2014-16

\begin{tabular}{|c|c|c|}
\hline Bias type & Description & Exploration/ adjustment of bias consequences \\
\hline $\begin{array}{l}\text { Survival / } \\
\text { Lead-time } \\
\text { bias }\end{array}$ & $\begin{array}{l}\text { More patients (69\%) in the intervention group than in the control group } \\
\text { (19\%) had Lead Days in the hospital before inclusion. } \\
\text { Mortality: Intervention patients must survive lead days to be referred and } \\
\text { included in PACT. Survivors may be healthier and cause a survival bias. } \\
\text { Sum emergency inpatient bed-days: In controls, we count "inpatient days" } \\
\text { from the first day of emergency admission. In PACT patients, we start } \\
\text { counting from the time of referral to PACT, leaving out emergency Lead } \\
\text { days before referral. If left unadjusted, this would bias comparisons towards } \\
\text { a lower sum of emergency days in intervention patients. }\end{array}$ & $\begin{array}{l}\text { Mortality risk analyses: } \\
\text { Restricted sub-group analyses to control-patients with a } \\
\text { survival time equal to or greater than median Lead-days } \\
\text { in the intervention group. } \\
\text { Sum emergency inpatient days: } \\
\text { We adjusted for Lead Days so that effect estimates are } \\
\text { independent of prior lead days. We tried matching on } \\
\text { lead-days, which would be the preferred avenue, but we } \\
\text { could not find enough matching controls for this. } \\
\text { We restricted analyses of sum emergency bed-days to } \\
\text { PACT patients with an index emergency hospitalisation } \\
\text { so that both groups add emergency bed-days from their } \\
\text { index episode to the 6-month outcome measure. }\end{array}$ \\
\hline
\end{tabular}

Indication Referral to the PACT intervention is less likely for terminal patients, or bias patients they judge to be unsuitable for the intervention for other reasons. In the control group, providers are likely to refer all other patients, including terminal patients to emergency admissions who then become eligible to be controls. We have no data, on the judgements made by referring professionals in either group.
Adjustment for possible under-referral of terminal patients to the intervention: We used the Elixhauser death risk score and the modified (m)-PARR30 score for both matching and adjustment. The C-statistic was 0,74 and 0,71 for death within six months in a local hospital population for these two predictors respectively. We made sub-group analyses restricted to controlpatients who survived median Lead-days to exclude terminal controls who died in their first few days in the hospital.

We estimated crude mortality risk in intervention patients that the PACT-team excluded since these might include terminal patients.

Sub-group analyses restricted to intervention patients whose index episode was an emergency admission.
Healthy $\quad 69 \%$ of the intervention and $100 \%$ of the control patient index episodes selection were linked to an emergency admission. Intervention patients who had no bias: index-episode emergency hospitalisation may be healthier than controls. 
abstract and results, we focus on multivariate-adjusted rate ratios $(\mathrm{RR}=$ Intervention Rate/ Control Rate) from the strictest sub-group analysis, as these represent our most unbiased effect estimates. We present results for other groups in Table 3.

The SNOW system $\odot$ [79] extracted data from the EHR for us. We used MatchIT v 2.4-21@ [70] for PS-matching. We used Stata $\odot$ v 14.0 and 15.0 for statistical analyses. We calculated heterogeneity $\mathrm{I}^{2}$ with "Comprehensive Meta-Analysis Version 3.0॰ [80].

\section{Results}

\section{Exclusions, balance between groups and study population}

530 persons were referred to the PACT-intervention for 606 care episodes from Oct. 2014 - Sept. 2016. The PACT-referral review team declined $5 \%$ of referrals. Figure 1 shows the exclusion flowchart. 83\% of all referred and 94\% of all eligible persons contributed to final analyses.
The dataset showed an excellent balance, with no balance tests outside of the recommended range. Some of the matching variables differed significantly between groups (see Table 2). As expected, the distribution of Lead days, which we were unable to match for, differs between groups.

\section{Hypothesis 1: reduction in emergency care utilisation}

All adjusted RR indicated a reduction of high-level emergency care utilisation.

In our strictest sub-group analysis, RR for emergency admissions was 0.90 (95\%CI: 0.82-0.99), RR for sum emergency bed days was 0.68 (95\%CI: $0.52-0.79)$ and RR for 30days readmissions 0.72 (95\%CI: 0.41-1.24). See Table 3 for details of multivariate analyses in other groups.

\section{Hypothesis 2: increased utilisation of low-level planned care}

The adjusted RR indicated a substantial increase in planned low-level care and no change in low-level emergency care.

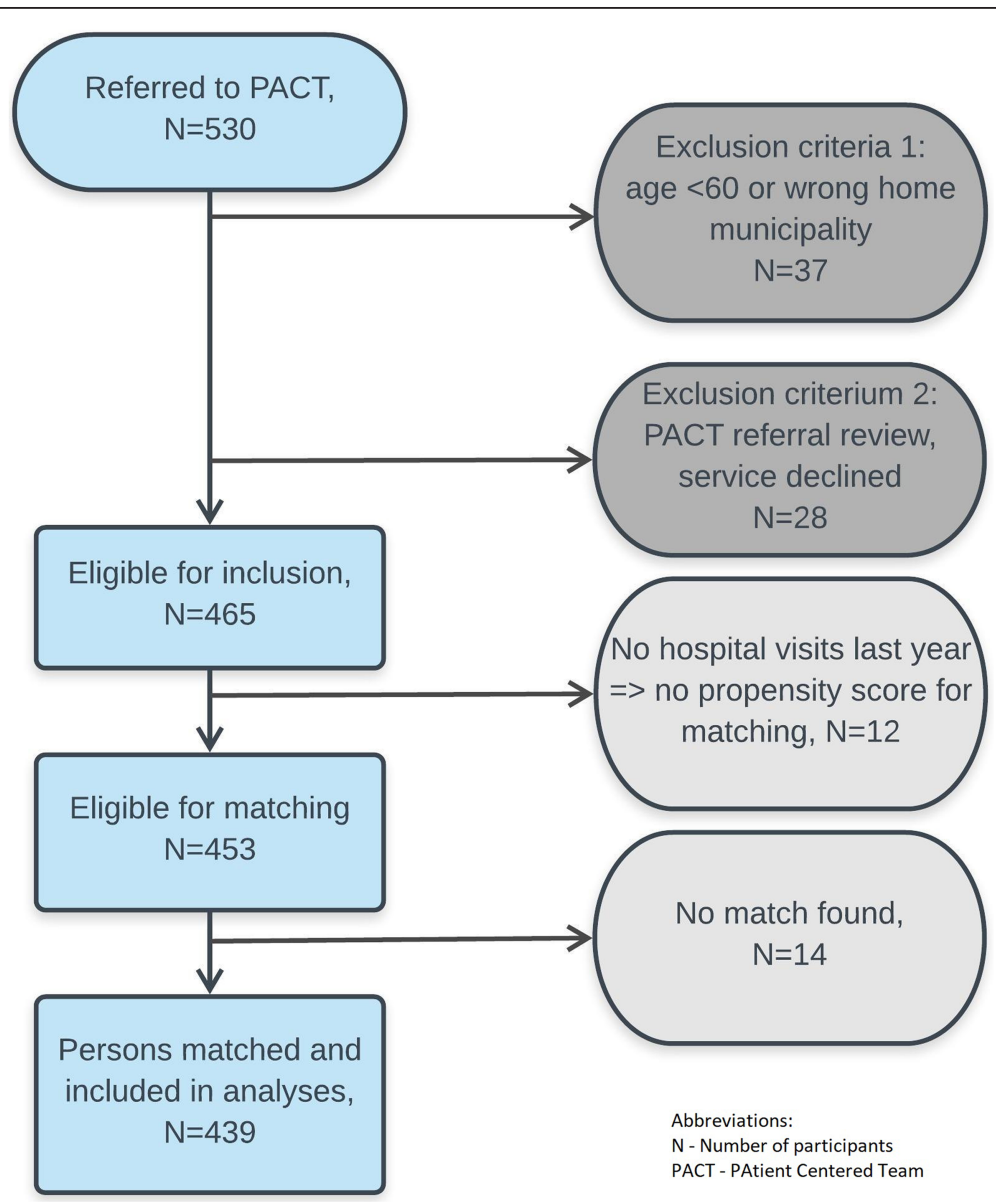

Fig. 1 Inclusion and exclusion Flowchart. Legend: The figure shows eligible PACT patients and exclusions at the person level. The Patient-Centred Team (PACT)-study, Norway 2014-16 
Table 2 Background variables and balance. Crude descriptive measures at baseline, for participants and their matched controls. If not otherwise marked, the point estimate is median and dispersion 5-95\%-tile. All variables in the table were matching variables, except for the two Lead Days variables. The Patient-Centred Team (PACT)-study, Norway 2014-16

\begin{tabular}{|c|c|c|c|c|c|c|c|c|}
\hline & \multirow[b]{2}{*}{ Unit } & \multicolumn{3}{|c|}{ Controls } & \multicolumn{3}{|c|}{ Intervention } & \multirow[t]{2}{*}{$p$} \\
\hline & & $\mathrm{N}$ & Point estimate & Dispersion & $\mathrm{N}$ & Point estimate & Dispersion & \\
\hline Sex (\%) & male & 779 & $41 \%$ & NA & 439 & $41 \%$ & NA & 0.51 \\
\hline Year at inclusion (\%) & in 2015 & 779 & $51 \%$ & NA & 439 & $51 \%$ & NA & 0.98 \\
\hline Age (mean/SD) & years & 779 & 78.81 & 8.68 & 439 & 80.02 & 8.72 & 0.02 \\
\hline m-PARR30. $2 Y$ (mean/SD) & Score & 779 & 2.19 & 0.57 & 439 & 2.16 & 0.61 & 0.49 \\
\hline DRG points. $1 Y$ & Sum & 779 & 2.20 & $0.03-12.65$ & 439 & 2.70 & $0.32-14.79$ & 0.10 \\
\hline \# Main diagnoses, $1 Y$ & Count & 779 & 3 & $1-8$ & 439 & 3 & $0-8$ & 0.30 \\
\hline \# Bi-diagnoses 1Y & Count & 779 & 3 & $0-13$ & 439 & 3 & $0-12$ & 0.05 \\
\hline \# Long-term Diagnoses. & Count & 779 & 11 & $2-29$ & 439 & 11 & $3-28$ & 0.41 \\
\hline m-PARR30, $2 Y$ & Score & 779 & 2.15 & $1.30-3.16$ & 439 & 2.09 & $1.33-3.20$ & 0.10 \\
\hline Elixhauser, 2Y & Score & 779 & 5 & $0-20$ & 439 & 5 & $0-20$ & 0.28 \\
\hline Emergency Inpt Adm. 1Y & Count & 779 & 2 & $0-8$ & 439 & 1 & $0-7$ & 0.05 \\
\hline Emergency Inpt Adm, 30d & Count & 779 & 1 & $0-4$ & 439 & 1 & $0-2$ & 0.96 \\
\hline Emergency Bed days, 30d & Sum & 779 & 2 & $0-15$ & 439 & 3 & $0-16$ & 0.03 \\
\hline Emergency Bed days, $1 Y$ & Sum & 779 & 6 & $0-52$ & 439 & 6 & $0-55$ & 0.86 \\
\hline Emergency Outpt visit, 30d & Count & 779 & 0 & $0-1$ & 439 & 0 & $0-1$ & 0.43 \\
\hline Emergency Outpt visit, 1Y & Count & 779 & 0 & $0-3$ & 439 & 0 & $0-3$ & 0.95 \\
\hline 30d Readmissions, $1 Y$ & Count & 779 & 0 & $0-1$ & 439 & 0 & $0-2$ & 0.26 \\
\hline Planned Inpt Adm, 1Y & Count & 779 & 0 & $0-4$ & 439 & 0 & $0-2$ & 0.04 \\
\hline Planned Inpt Adm 30d & Count & 779 & 0 & $0-1$ & 439 & 0 & $0-1$ & 0.74 \\
\hline Planned Outpt visit, $1 Y$ & Count & 779 & 2 & $0-21$ & 439 & 2 & $0-18$ & 0.22 \\
\hline Planned Outpt visit, 30D & Count & 779 & 0 & $0-3$ & 439 & 0 & $0-3$ & 0.06 \\
\hline Lead Days & Count & 779 & 0 & $0-9$ & 439 & 4 & $0-30$ & $<0.001$ \\
\hline Quintile Lead Days (\%) & Q $1+2$ & 779 & $78 \%$ & NA & 439 & $20 \%$ & NA & $<0.001$ \\
\hline
\end{tabular}

Abbreviations: $N$ Number of patients, \# Number of, p-probability, SD Standard deviation, m-PARR30 modified PARR score [76], Elixhauser score - Elixhauser Comorbidity Measure [77], DRG Diagnosis Related Groups [64], Readmissions - New emergency admission within 30 days of last hospital discharge, Inpt Inpatient, Adm Admission, Outpt Outpatient. Main-diagnosis: The current diagnosis which caused admission. Bi-diagnoses: Other diagnoses relevant for the care of the current problem

Time spans: $1 \mathrm{Y}$ - Last year prior, $2 \mathrm{Y}$ - Last 2 years prior, $30 \mathrm{~d}$ - Last 30 days prior

Statistics: $P$-values for two-sided t-tests (continuous normal variables) Wilcoxon rank-sum test (continuous non-normal variables) and Chi2-test (discrete variables) for difference between control and intervention groups

The RR for planned outpatient visits was 2.3 (95\%CI: 2.02-2.55), while RR for emergency outpatient visits was 0.9 (95\%CI: $0.68-1.20)$ in our strictest sub-group analysis. Please see Table 3 for details of multivariate analyses in other groups.

\section{Hypothesis 3: unchanged mortality rates}

The adjusted RR indicated a reduction in mortality risk at both three and six months follow-up.

In all, $74(17 \%)$ and $180(23 \%)$ patients died within the 6-month follow-up in the intervention and control group respectively. The median survival time in both the control and intervention groups was 182.5 days (5-95\%-tile Controls: 12-182.5, PACT: $35-182.5, p=0.009$ ).
The RR for death at three months was 0.39 (95\% CI: $0.22-0.70$ ) in our strictest sub-group analysis. The protection waned somewhat after three months so that at six months the RR was 0.57 (95\% CI: 0.34-0.94), also in our strictest sub-group analysis. Please see Table 3 for details of multivariate analyses for other groups. The Kaplan-Meier plot shows a net survival benefit in the intervention groups by the end of the follow-up period (Fig. 2).

There were in all 28 referred patients who did not get the PACT intervention within $24 \mathrm{~h}$ and were therefore ineligible for analyses. Of these 6 (21\%) died. There were in all $4(15 \%)$ deaths among the 26 persons who did receive the intervention but were excluded from analyses due to technical reasons (no prior hospital history, no suitable match). Small numbers preclude drawing any conclusion from these numbers. 
Table 3 Rate Ratios for outcomes: Multivariate multilevel adjusted pooled analysis, at six months follow-up, by outcomes and subgroup. Negative Binomial regression for health care utilisation outcomes and Cox regression for mortality. The Patient-Centred Team (PACT)-study, Norway 2014-16

\begin{tabular}{|c|c|c|c|c|c|c|}
\hline \multirow[t]{2}{*}{ Outcome } & \multirow[t]{2}{*}{ Population sub-groups } & \multirow[t]{2}{*}{$\mathrm{N}$} & \multirow[t]{2}{*}{ RR } & \multirow[t]{2}{*}{$p$} & \multicolumn{2}{|l|}{$95 \% \mathrm{Cl}$} \\
\hline & & & & & Lower & Upper \\
\hline \multirow{4}{*}{$\begin{array}{l}\text { Count, Emergency Admissions after } \\
\text { index episode }\end{array}$} & All, adjusted & 1218 & 0.95 & $<0.001$ & 0.94 & 0.96 \\
\hline & Only controls surviving Lead days (1) & 1195 & 0.95 & 0.024 & 0.91 & 0.99 \\
\hline & $\begin{array}{l}\text { Only PACT patients with index emergency } \\
\text { hospitalizations (2) }\end{array}$ & 856 & 0.90 & 0.018 & 0.82 & 0.98 \\
\hline & Combination of 1 and 2 & 838 & 0.90 & 0.033 & 0.82 & 0.99 \\
\hline \multirow[t]{2}{*}{$\begin{array}{l}\text { Sum, Emergency Bed days (including } \\
\text { index episode bed days) }\end{array}$} & $\begin{array}{l}\text { Only PACT patients with index emergency } \\
\text { hospitalizations (2) }\end{array}$ & 856 & 0.62 & $<0.001$ & 0.49 & 0.77 \\
\hline & Combination of 1 and 2 & 838 & 0.68 & 0.005 & 0.52 & 0.89 \\
\hline \multirow{4}{*}{$\begin{array}{l}\text { Count, emergency readmissions within } \\
30 \text { days of discharge, after index episode }\end{array}$} & All, adjusted & 1218 & 0.64 & $<0.001$ & 0.52 & 0.78 \\
\hline & Only controls surviving Lead days(2) & 1195 & 0.63 & $<0.001$ & 0.51 & 0.79 \\
\hline & $\begin{array}{l}\text { Only PACT patients with index emergency } \\
\text { hospitalizations ( } 2 \text { ) }\end{array}$ & 856 & 0.71 & 0.213 & 0.41 & 1.22 \\
\hline & Combination of 1 and 2 & 838 & 0.72 & 0.231 & 0.41 & 1.24 \\
\hline \multirow[t]{4}{*}{ Count, Planned outpatient visits } & All, adjusted & 1218 & 2.40 & $<0.001$ & 2.21 & 2.61 \\
\hline & Only controls surviving Lead days(1) & 1195 & 2.41 & $<0.001$ & 2.22 & 2.62 \\
\hline & $\begin{array}{l}\text { Only PACT patients with index emergency } \\
\text { hospitalizations (2) }\end{array}$ & 856 & 2.26 & $<0.001$ & 2.01 & 2.54 \\
\hline & Combination of 1 and 2 & 838 & 2.27 & $<0.001$ & 2.02 & 2.55 \\
\hline \multirow[t]{4}{*}{ Count, Emergency Outpatient visits } & All, adjusted & 1218 & 0.82 & 0.001 & 0.73 & 0.92 \\
\hline & Only controls surviving Lead days(1) & 1195 & 0.82 & $<0.001$ & 0.76 & 0.88 \\
\hline & $\begin{array}{l}\text { Only PACT patients with index emergency } \\
\text { hospitalizations (2) }\end{array}$ & 856 & 0.89 & 0.408 & 0.67 & 1.18 \\
\hline & Combination of 1 and 2 & 838 & 0.90 & 0.464 & 0.68 & 1.20 \\
\hline \multirow[t]{4}{*}{ Mortality 0-3 months } & All, adjusted & 1218 & 0.38 & $<0.001$ & 0.24 & 0.60 \\
\hline & Only controls surviving Lead days(1) & 1195 & 0.46 & 0.001 & 0.28 & 0.73 \\
\hline & $\begin{array}{l}\text { Only PACT patients with index emergency } \\
\text { hospitalizations (2) }\end{array}$ & 856 & 0.32 & $<0.001$ & 0.19 & 0.55 \\
\hline & Combination of 1 and 2 & 838 & 0.39 & 0.001 & 0.22 & 0.70 \\
\hline \multirow[t]{4}{*}{ Mortality 0-6 months } & All, adjusted & 1218 & 0.53 & 0.001 & 0.37 & 0.77 \\
\hline & Only controls surviving Lead days(1) & 1195 & 0.60 & 0.010 & 0.41 & 0.89 \\
\hline & $\begin{array}{l}\text { Only PACT patients with index emergency } \\
\text { hospitalizations (2) }\end{array}$ & 856 & 0.48 & 0.003 & 0.30 & 0.78 \\
\hline & Combination of 1 and 2 & 838 & 0.57 & 0.028 & 0.34 & 0.94 \\
\hline
\end{tabular}

Abbreviations: $N$ Number of patients, RR Rate Ratio (Rate Interv /Rate Control), $p$ probability, 95\% Cl 95\% confidence interval

Sub-group analyses: (1) Only controls surviving Lead days: Controls who survived the intervention group's median lead days in the hospital. Excluded: Controls who died during the first 4-5 days and their matches. (2) Only intervention patients with an index emergency hospital episode. Excluded: Intervention patients with index episode in the municipality or planned hospitalisation and their matches

Final model adjustment variables: Emergency admissions: Fixed effect: Count of emergency admissions last year, Site. Random effect: site, triplet-stratum ID. Final model adjustment variables: Sum emergency inpatient days: Fixed effect: Quintile of lead days, Sum emergency bed days last year, Site. Random effect: site, triplet-stratum ID

Final model adjustment variables: Count 30-day Readmissions: Fixed effect: Quintile of lead days, Count re-admissions last year, Site. Random effect: site, tripletstratum ID.

Final model adjustment variables: Planned outpatient visits: Fixed effect: Count planned outpatient visits last year, Site. Random effect: site, triplet-stratum ID Final model adjustment variables: Emergency outpatient visits: Fixed effect: Quintile lead days, Count emergency outpatient visits last year, Site. Random effect: site, triplet-stratum ID

Final model adjustment variables: Mortality 0-3 months: Fixed effects: Quintile lead days, Age, Elixhauser score, Site. Random effect: site, triplet-stratum ID Final model adjustment variables: Mortality 0-6 months: Fixed effects: Quintile lead days, Count readmission last year, Age, Elixhauser score, Site. Random effect: site, triplet-stratum ID 


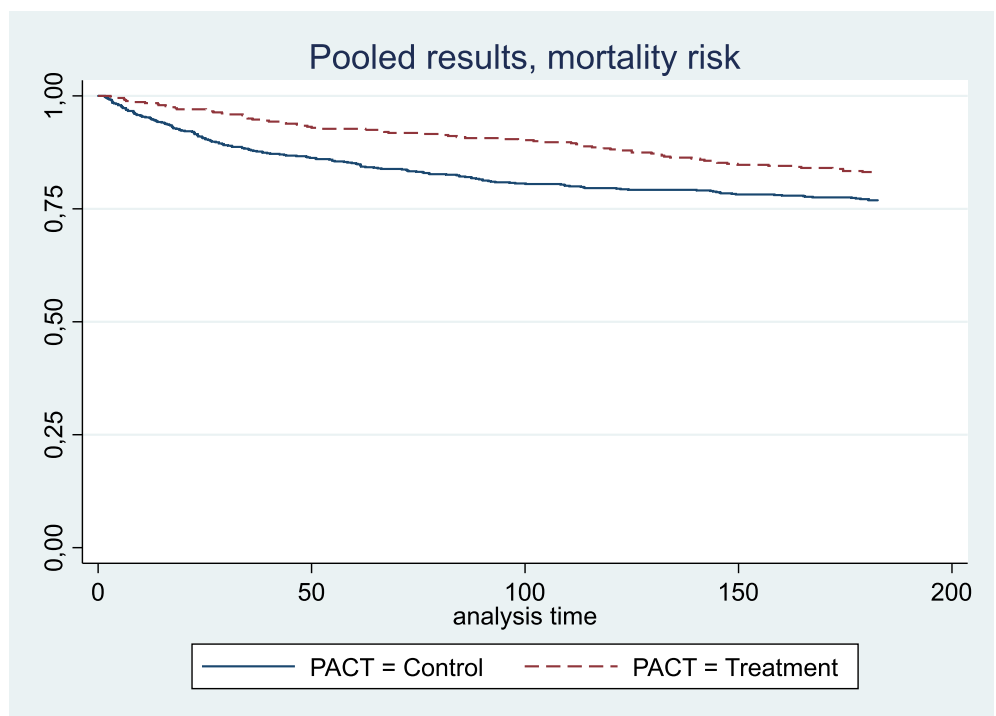

Fig. 2 Mortality. Legend: Crude Kaplan-Meier curves, showing the proportion of patients alive by time, and group in 6 months follow-up period. Pooled data, ( $N=1218)$. The Patient-Centred Team (PACT)-study, Norway 2014-16

\section{Sub-group and sensitivity analyses}

\section{Survival and indication bias}

When we compared RRs of "All, adjusted" with that of "Only controls surviving Lead days" we saw a weakening of the three month RR mortality from 0 , 38 to $0,46(\Delta 16 \%)$ and six month RR from 0,53 to 0 , $66(\Delta 11 \%)$ (Table 3$)$. The result implies survival and indication bias, but the intervention effect was still substantial. A Kaplan Meier plot of the strictest subgroup, excluding outlier sites also showed a net benefit for the intervention group. (See Additional file 2) No other outcome changed $>10 \%$.

\section{Healthy selection bias}

We saw a strengthening of the three month RR estimate from 0,38 to $0,32(\Delta 19 \%)$ when comparing RRs for "All" with "Only index emergency hospitalisations" (Table 3 ). The finding weakens the case for healthy selection bias. No other outcome changed $>10 \%$.

\section{Lead time bias}

The crude RR estimate of sum-emergency days changed direction from a crude RR of 1.1 to an adjusted RR of 0.58 upon adjustment for Lead days, showing Lead days to be a strong confounder for this outcome (data not shown).

\section{Heterogeneity and interactions with site}

There was no evidence of interaction across intervention sites, as the interaction term between intervention site and outcome were not significant for any outcomes (data not shown). Except for 30day readmissions, all outcomes displayed significant heterogeneity across control sites, but the control sites took turns in being the outlier for each outcome (See Additional file 2). Exclusion of outlier sites changed the 6 month mortality RR point estimates for one sub-group analysis: "Only index emergency hospitalisations" from a RR of $0,48(p=0$, $003)$ to a RR of $0,54(\mathrm{p}=0,01)(\Delta 11 \%)$. No other outcomes changed $>10 \%$.

\section{Discussion}

\section{Principal findings}

Compared with propensity score matched controls, the care process of frail multi-morbid elderly who received the PACT intervention had a reduced risk of high-level emergency care, increased use of low-level planned care, and substantially reduced mortality risk.

\section{Strengths and weaknesses Internal validity}

As administrative personnel was unaware of the treatment status, systematic misclassification of outcomes is unlikely. The high degree of completeness of data makes missing outcomes a negligible problem [65], and strengthens internal validity.

Bias evaluation: We have discussed some possible biases that might have influenced our analysis. Admittedly, analyses restricted to controls who had survived the median lead-time showed a weaker, albeit still significant mortality risk reduction. It is relevant to point out that this sub-group analysis is, an "over-correction" because by definition half of the intervention patients will have survived a shorter lead-time than the median, whereas all the controls survived the lead-time period. The strictest sub-group analysis, combining adjustment 
for both indication and healthy selection bias, did not erase our findings.

We are naturally unable to account for unknown biases or confounding, as they would have been in an RCT. We have no systematic review of referral practices. The care process documentation is limited to changes in the health care utilisation outcomes, which did support our hypotheses, although a more detailed process evaluation is called for. Both challenges are limitations, and areas for future research (see below).

\section{External validity}

Non-parametric pre-processing by use of propensity score matching allows a post hoc creation of a control group and reduces model dependencies [81]. Although considered to have a lower internal validity than an RCT, its applicability to "real world" data gives a higher external validity [82]. The balance measures were excellent, warranting comparability between groups on all known variables. The matching software program (MatchIt) had no access to follow-up data, which ensured that matching was not influenced by outcomes data, which is a critique towards this methodology [83].

Since we were granted permission to extract routine data without formal consent, we were able to include both patients with cognitive impairment and/ or acute morbidity. Both conditions contribute to the underrepresentation of elderly in clinical research [84]. Exclusions of these highly vulnerable patients from outcomes evaluations, represent an ethical challenge, as these populations have an equal right to quality assurance and evaluation of care as other patient groups [84].

Norwegian health care constitutes its own context, which probably differs from that of other countries in important respects. We have one study that shows low integration of care in Norway [40]. The context dependency is an area worthy of further research (see below). Within Norway, we implemented the intervention at two sites and found no interactions by Site. Effects did vary by control-context, but the intervention effect was significant even after we had adjusted for control-site variation as a random effect. The study has high external validity, which is a strength.

\section{PACT results in light of literature}

\section{PACT compared to other goal-oriented PCC interventions}

The Goal-oriented PCC care of PACT has still no internationally recognised vocabulary. However, similar approaches are "flipped care" [30], and "personalised care planning" [15]. Their commonality is the construction of personalised goals together with the patient, which subsequently drives care planning, care delivery, and care evaluation. "What matters" is the organising principle of care, inescapably present throughout the entire care process. Our PCC approach differs fundamentally from other person-centred approaches linked to integrated care, where authors often describe tailoring of care plans to personal values, needs, and preferences, yet they fail to show how plans, care delivery and evaluation become shaped by the person's goals [15].

We have found only one controlled clinical trial directed at persons with CLNs which applies "flipped healthcare" similarly to us. Sweeney compared a "Patient-Centred management" with usual case-management for cancer patients at the end of life and found reduced care utilisation and costs, with no shortening of life [85]. Coulter's Cochrane review on personalised care planning reports a small but significant improvement in bio-psycho-medical health, which "... appear greater when the intervention is more comprehensive, more intensive, and better integrated into routine care." However, she found no eligible studies on multi-morbidity in the review, which makes her promising findings tangential to our work. Two protocols that subscribe to a goaloriented PCC care process show that Goal-PCC is an up and coming concept $[86,87]$.

\section{PACT compared to interventions aligned with the chronic care model}

We also compared PACT with comprehensive care interventions directed at persons with CLNs, defined as at least two components of the Chronic Care Model (CCM) [16]. Of 15 studies that reported mortality risk, 3 reduced mortality significantly [88-90]. Two of the three studies are from Norway $[89,90]$. In a comparative study across 11 nations on gaps in care integration, Norway performed poorly [40]. As there is a lack of follow-up studies, at this stage we can only speculate on whether this is why the Norwegian context seems to be more responsive to comprehensive care interventions. However, it is difficult to see any clear pattern of how the three positive studies differ from the 12 negative studies. de Bruin concluded in her review that "No evidence was found for a beneficial effect of comprehensive care on ( ...) mortality." de Bruin also notes the diversity in effects from comprehensive care programs, and links it to the variation in interventions and context [16].

We found two other studies that resembles the PACT study, in that they apply a three-component PCC, integrated and pro-active care model. Yet neither of these had paid close attention to the synergies between the three elements. Neither study showed any effects on hospital health care utilization, costs or function. This is in line with several recent reviews of multi-component comprehensive care models designed to address the challenges of multi-morbidity or frail patients, where the results across studies are mixed [21, 22, 91-93]. The reviews lament the heterogeneity of both target groups 
and intervention designs. We believe a careful untangling of the desired goals, and the chain of causes and effects that lead to the desired goal at both population and individual levels are not yet well enough understood.

\section{PACT mechanisms for effect}

The PACT intervention did not introduce any new biological treatments. The mechanisms for a mortality difference between the groups must either be the result of bias between the groups, the result of the intervention or a combination of both. While we have discussed possible biases at great length above, we here present some of the mechanisms of the intervention, which we hypothesise, might contribute to our results.

\section{- Patient detected quality challenges: The} continuous dialogue with the patient on "what matters to you" give insights into challenges that only the patient can detect, and allows the team to address them effectively. Examples are gaps in care, where delivery of planned care failed, such as missing referrals or missing prescriptions, adverse events that could influence patient willingness to follow self-management advice, i.e. a hypoglycemic episode following recommended exercise, or ineffective pain management, which hinders mobility training.

- Proactive care planning: Comprehensive geriatric assessment includes an early evaluation and management of common geriatric complaints, such as exercises in sarcopenia, and effective undernourishment management [63]. We added several complementary risk-management strategies, (see intervention design) designed to stabilise the health situation and prevent further clinical crises.

- Avoid iatrogenic disability: frail elderly are vulnerable, at risk for a large fall in function following minor insults [63]. Hospitalization of elderly patients is associated with delirium, decreased mobility and increased dependency [94]. PACT works to mitigate decompensation through early discharge to a familiar environment.

- Prescription review: performed by the pharmacist revealed errors and interactions in a majority of the medication lists reviewed. The pharmacist corrected such errors in collaboration with the relevant professionals.

- Improved care delivery according to plan: Due to fragmented information and organisational systems, professionals have few tools to support the interdisciplinary dialogue and collaboration. Typically, no professional will have the full overview of the needs or activities for patients with CLNs.
Gaps and overlaps are virtually "invisible" in a silobased system. The PACT team has the time and capacity to make an overview of all care, facilitate dialogue across organisations and professions and continuously update all parties involved in the care plan. PACT ensures that the care plan aligns with the patient's personal goals, planned activities are not at odds with one another, and care plans translate into actual care delivery.

- Professional motivation: The goal-oriented PCC serves to make all parties work towards the same goal. It not only makes sense to the person who needs care. Working together with the patient towards "What matters" seems to have a substantial motivational effect on involved professionals across organisations in the primary and second care sector [32]. PACT team members feel they are "making a difference", which may in itself influence outcomes.

- Flexible and adaptive goal driven care: Finally, PACT does not force care planning into a specific method or format. Instead, the agents follow a set of principles and pragmatically apply them to reach the negotiated goals. We hypothesise that this flexibility allows the partners to create tailored solutions, which may be more effective than multiple uncoordinated standardised pathways for multi-morbid patients [26].

PACT builds on real-world data, has shown transferability across two intervention sites, and produce consistent results across control sites. The PACT project aimed to remedy universally acknowledged challenges [1-4]. Our health care utilisation measures show that PACT succeeds in reducing the emergency care and increase planned low-level planned care. Our underlying hypotheses for why our intervention works are in accord with the literature [9-14]. To summarise, the substantial effects of the "real world" PACT study, concerning both mortality and health service utilisation is unusual.

\section{Implications for practice and further research}

Several comparable interventions in the literature proactively identify frail patients in GP EHR, but these studies fail to show effects $[95,96]$. It may be, that it is not enough to capture frailty, as some very frail persons may be balancing their lives well enough, and interventions may upset their precarious balance. Finding formal methods of capturing the frail person as they move to an unstable state, while there is still time to regain balance, is an important research question. A formal definition of the PACT target population would also allow us to evaluate how outcomes for a formally identified frail elderly in intervention municipalities compared to the same group in control municipalities. 
To further examine external validity in an international context, a comparison of the Norwegian results with international propensity score matched controlgroups is an aim for future research.

Although we attribute effects to our theoretical underpinnings, the study merits further evaluation of the causes of death in the two treatment groups, to understand better how effects are produced [97]. A stronger understanding of the care process and mechanisms of effect and how patient pathways differ between groups and contexts would help us understand which components require high fidelity in any intervention site, and which components can and should tailor to the local context.

\section{Conclusions}

The PACT intervention lowers the need for high-resource emergency care, increases use of planned low-resource care and protects vulnerable patients from death compared to propensity score matched controls. We have used a robust methodology and have adequate statistical power to support our findings. The PACT study has designed a person-centred, integrated and pro-active care model, which attends to, responds to and is loyal to "what matters to you?", using the cyclical goal-oriented adaptive solving strategies from complexity theory. We argue that our study is a contribution towards a better understanding of care for persons with CLNs. Further studies of the effects of goal-oriented PCC may force the highly needed paradigm shift that will make genuine goal driven PCC the norm.

\section{Additional files}

Additional file 1: The PACT care model. A stronger and more detailed qualitative description of the PACT intervention. (DOCX $210 \mathrm{~kb}$ )

Additional file 2: Supplementary analyses. This file includes additional tables showing: Crude results for all outcomes. Pooled adjusted results for all outcomes, showing 12 (the heterogeneity index across sites), with outlier-sites first included and then excluded. The Kaplan-Meier plot for mortality, with outlier sites excluded. (DOCX 53 kb)

\section{Abbreviations}

CLN: Long-term and Complex Needs; DRG: Diagnosis Related Group; EHR: Electronic Health Record; Goal-PCC: Goal oriented Person-Centred Care; IC: Integrated Care; KM: Kaplan Meier plot; Lead Days: \# of days in hospital leading up to inclusion in the study; M1 and M2: Municipality 1 and Municipality 2; MD: Mahalanobis Distance; m-PARR30: modified PARR30 score, UK specific coefficients omitted; NPR: Norwegian Patient Registry; PACT: The PAtient Centred Team; PCC: Person-Centred Care; PH: Proportional Hazards; Pro-C: Proactive Care; PS: Propensity Score; REC: Regional Ethics Committee; RR: Rate Ratio

\section{Acknowledgements}

Not applicable.

\section{Authors' contributions}

All authors contributed to the idea of the paper and critically reviewed the drafts, provided feedback and approved the final manuscript. GB performed the statistical analyses, wrote the drafts, and circulated and revised the manuscript according to input. JH and JGB extracted data from the EHR and performed matching. GB, SOS, and TB1 provided statistical and analytical expertise. MD, BS, LS, TB2, and MR were instrumental in the design and implementation of the PACT intervention. The implementation builds on insights gained from patients, published elsewhere [31, 41]. All authors, external and internal, had full access to all of the analytic results (including statistical reports and tables) in the study, can take responsibility for the integrity of the accuracy of the data analysis, and have read and approved the manuscript. Transparency declaration: GB is the guarantor of the study. GB affirms that the manuscript is an honest, accurate, and transparent account of the study; that no important aspects of the study have been omitted; and that any discrepancies from the study as planned have been explained.

\section{Funding}

We gratefully acknowledge the contribution from our funders: The Regional health authorities in Norway, the contributions regarding financial and personnel resources to implement the intervention from Troms $\varnothing$ and Harstad municipalities, the medical clinics and the Norwegian center for ehealth research at the University Hospital of North-Norway. All authors have completed the Unified Competing Interest form and declare that this work has been funded by two research grants from the Regional Health Authorities of northern Norway's research fund grants: \# HST1243-15 and \#HST1242-15, and one grant from the research fund HELSE-FORSK,\# 243857. This research has been undertaken with full independence from funders. We have no financial relationships with any organisations that might have an interest in the submitted work in the previous three years, no other relationships or activities that could appear to have influenced the submitted work.

\section{Availability of data and materials}

Due to strict Norwegian privacy, data cannot be shared openly. Data can be made available for external researchers if approval from the Norwegian Regional Ethics Committee (REC) is obtained. Please contact the corresponding author for assistance to apply for an approval from REC. REC can be also contacted directly regarding the project's approvals (\#2014/1707/REK Sør-Øst A).

\section{Ethics approval and consent to participate}

Patients enrolled in the PACT service had to provide an informed oral consent, documented in the Electronic Health Record (EHR), to receive the clinical PACT services. In the case of no consent, the patient would receive usual clinical care. The first patient was enrolled into the PACT service in October 2014.

The Regional Ethics committee (REC) (\#2014/1707/REK Sør-Øst A), found the intervention itself to be exempt from Ethics review.

Even when REC does not review the intervention, REC is by law required to process requests to use routine EHR data for research without consent from patients. Due to high acute morbidity, challenging and overwhelming life situations, and a high proportion of patients with cognitive impairments, the recruitment of PACT patients into the evaluation study was slow. We therefore sought and was granted REC approval for the extraction of patient EHR data, without patient consent, under § 7-26 in the Personal Information Regulation, and the Health-provider Act $\$ 26$. This approval was provided in October 2015.

The privacy ombudsman approved the privacy and information security aspects of the study at the Univ hospital of North Norway (\# 0547) in November 2015. Steps taken to ensure privacy protection were: the analytic files were de-identified, i.e. all direct identification variables had been replaced by a pseudonym. Analysis files were saved and analysed on "offline" devices/ computers. Only 3 researchers $(G B, J H, T B 1)$ had access to the analysis files. All intermediate and final results were fully anonymized. We report here on secondary outcomes extracted from the EHR: Mortality and health services utilization. The first EHR data were extracted in Jan 2016 after we had obtained necessary formal approvals from REC and the privacy ombudsman.

Consent to publish from individual participant data is not applicable to this paper.

We continue a consent based collection of Patient reported outcome measures. We enrolled the first patient for PROMs in Nov 2015, which is our primary outcome according to the published protocol. PROMs collection will 
continued until march 2019. These results will be published later in a separate paper.

\section{Consent for publication}

Not applicable

\section{Competing interests}

The authors declare that they have no competing interests.

\section{Author details}

${ }^{1}$ Norwegian Center for e-health research, University Hospital of North Norway Trust, Universitetssykehuset, PB 35, 9038 Tromsø, Norway. ${ }^{2}$ Clinic of general medicine, University Hospital of North Norway Trust, Universitetssykehuset, PB 100, 9038 Tromsø, Norway. ${ }^{3}$ Clinic of general medicine, University Hospital of North Norway Trust, Harstad hospital, PB 1065, 9480 Harstad, Norway. ${ }^{4}$ Director of Health and Care, Troms Municipality, PB 6900, Tromsø 9299, Norway. ${ }^{5}$ Institute of community medicine, UiT The Arctic University of Norway, PO Box 6050 Langnes, N-9037 Tromsø, Norway.

Received: 12 September 2018 Accepted: 1 August 2019 Published online: 03 October 2019

\section{References}

1. Tinetti ME, Fried T, Boyd C. Designing health care for the most common chronic condition-multimorbidity. JAMA. 2012;307:2493-4

2. Bayliss EA, Edwards AE, Steiner JF, Main DS. Processes of care desired by elderly patients with multimorbidities. Fam Pract. 2008;25:287-93.

3. Wallace E, Salisbury C, Guthrie B, Lewis C, Fahey T, Smith SM. Managing patients with multimorbidity in primary care. BMJ. 2015:350:1-6.

4. Vogeli C, Shields AE, Lee TA, Gibson TB, Marder WD, Weiss KB, Blumenthal $D$, Vogeli $C$, Shields $A E$, Lee TA, et al. Multiple chronic conditions: prevalence, health consequences, and implications for quality, care management, and costs. J Gen Intern Med. 2007;22(Suppl 3):391-5.

5. Heiberg I. High utilisation patients in somatic specialist healthcare in Northern Norway. [Storforbrukere av somatisk spesialisthelsetjeneste i Helse Nord] [https://helse-nord.no/Documents/SKDE/SKDE\%20Publikasjoner/ Storforbrukere\%20av\%20somatisk\%20spesialisthelsetjeneste\%20i\%2 OHelse\%20Nord\%20(SKDE\%202015).pdf] Accessed on date: 2015-12-06

6. Zulman DM, Pal Chee C, Wagner TH, Yoon J, Cohen DM, Holmes TH, Ritchie C, Asch SM. Multimorbidity and healthcare utilisation among high-cost patients in the US Veterans Affairs Health Care System. BMJ Open. 2015;5(4):1-9.

7. Wang L, Si L, Cocker F, Palmer AJ, Sanderson K. A Systematic Review of Cost-of-Illness Studies of Multimorbidity. Appl Health Econ Health Policy. 2017;16:15-29.

8. Berwick DM, Nolan TW, Whittington J. The Triple Aim: Care, Health, And Cost. Health Aff. 2008;27(3):759-69.

9. Valentijn PP, Vrijhoef HJM, Ruwaard D, Boesveld I, Arends RY, Bruijnzeels MA. Towards an international taxonomy of integrated primary care: a Delphi consensus approach. BMC Fam Pract. 2015;16:1-15.

10. Nolte E, McKee M. Caring for people with chronic conditions: a health systems perspective [http://www.euro.who.int/_data/assets/pdf_file/0006/ 96468/E91878.pdf] Accessed on date: 8 Aug 2019

11. Muth C, van den Akker M, Blom J, Mallen C, Rochon J, Schellevis F, Becker A, Beyer M, Gensichen J, Kirchner H, et al. The Ariadne principles: how to handle multimorbidity in primary care consultations. BMC Med. 2014;12:1-11.

12. Multimorbidity: Clinical Assessment And Management. Multimorbidity: assessment, prioritisation and management of care for people with commonly occurring multimorbidity. In: Secondary Multimorbidity: clinical assessment and management. UK: National Institute for Health and Care Excellence; 2016. p. 1-443. Edited by Secondary.

13. Rich E, D. L, J L, M. P. Coordinating Care for Adults With Complex Care Needs in the Patient-Centered Medical Home: Challenges and Solutions. White Paper [Archived at: http://www.webcitation.org/73DWky5eg] Accessed on date: 8 Aug 2019

14. Smith SM, Cousins G, Clyne B, Allwright S, O'Dowd T. Shared care across the interface between primary and specialty care in management of long term conditions. Cochrane Libr. 2017:2:1-143.

15. Coulter A, Entwistle Vikki A, Eccles A, Ryan S, Shepperd S, Perera R. Personalised care planning for adults with chronic or long-term health conditions. Cochrane Database Syst Rev. 2015;3:1-129.
16. de Bruin SR, Versnel N, Lemmens LC, Molema CCM, Schellevis FG, Nijpels G, Baan CA. Comprehensive care programs for patients with multiple chronic conditions: A systematic literature review. Health Policy. 2012;107(2-3):108-45.

17. Lemmens LC, Molema CCM, Versnel N, Baan CA, de Bruin SR. Integrated care programs for patients with psychological comorbidity: A systematic review and meta-analysis. J Psychosom Res. 2015;79(6):580-94.

18. Vegesna A, Tran M, Angelaccio M, Arcona S. Remote patient monitoring via non-invasive digital technologies: a systematic review. Telemed e-Health. 2017;23(1):3-17.

19. Foster G, Taylor S, Eldridge S, Ramsay J, Griffiths C. Self-management education programmes by lay leaders for people with chronic conditions (Review); 2009. p. 1-78.

20. WHO. People-centred and integrated health services: an overview of the evidence. Interim report. In: Secondary People-centred and integrated health services: an overview of the evidence. Interim report. Geneva: WHO; 2015. p. 1-65. Edited by Secondary.

21. Flanagan S, Damery S, Combes $G$. The effectiveness of integrated care interventions in improving patient quality of life $(\mathrm{OoL})$ for patients with chronic conditions. An overview of the systematic review evidence. Health Qual Life Outcomes. 2017;15:1-11.

22. Smith SM, Wallace E, O'Dowd T, Fortin M. Interventions for improving outcomes in patients with multimorbidity in primary care and community settings. Cochrane Libr. 2016;3:1-115.

23. Steventon A, Bardsley M, Billings J, Dixon J, Doll H, Hirani S, Cartwright M, Rixon L, Knapp M, Henderson C. Effect of telehealth on use of secondary care and mortality: findings from the Whole System Demonstrator cluster randomised trial. BMJ. 2012:344:1-15.

24. McBain H, Shipley M, Newman S. The impact of self-monitoring in chronic illness on healthcare utilisation: a systematic review of reviews. BMC Health Serv Res. 2015;15:1-10.

25. Valentijn PP, Schepman SM, Opheij W, Bruijnzeels MA. Understanding integrated care: a comprehensive conceptual framework based on the integrative functions of primary care. Int J Integr Care. 2013:13(1):1-12.

26. Reed JE, Howe C, Doyle C, Bell D. Simple rules for evidence translation in complex systems: A qualitative study. BMC Med. 2018:16(1):1-20.

27. WHO. Framework on integrated, people-centred health services. Report by the Secretariat [Accessed at: http://apps.who.int/gb/ebwha/pdf files/WHA6 9/A69_39-en.pdf?ua=1 ] Accessed on date: 8 Aug 2019

28. Mathers $\mathrm{N}$, Paynton D. Rhetoric and reality in person-centred care: introducing the House of Care framework. Br J Gen Pract. 2016;66(642):12-3.

29. Person-centered care made simple [Archived at: http://www.webcitation. org/73DX9aYtT] Accessed on date: 8 Aug 2018

30. Bisognano M, Schummers D. ESSAY Flipping healthcare: an essay by Maureen Bisognano and Dan Schummers. BMJ. 2014;349:1-3.

31. Berntsen GKR, Gammon D, Steinsbekk A, Salamonsen A, Foss N, Ruland C, Fønnebø V. How do we deal with multiple goals for care within an individual patient trajectory? A document content analysis of health service research papers on goals for care. BMJ Open. 2015;5:1-8.

32. Miller E, Stanhope V, Restrepo-Toro M, Tondora J. Person-centered planning in mental health: A transatlantic collaboration to tackle implementation barriers. Am J Psychiatr Rehabil. 2017;20(3):251-67.

33. Murante AM, Seghieri C, Vainieri M, Schäfer WL. Patient-perceived responsiveness of primary care systems across Europe and the relationship with the health expenditure and remuneration systems of primary care doctors. Soc Sci Med. 2017;186:139-47.

34. Ringard Å, Sagan A, Saunes IS, Lindahl AK. Norway: Health system review. Health Systems in Transition. Health Syst Transit. 2014;15(8):1-162.

35. OECD: Health at a Glance: Europe. State of health in the EU Cycle. In: Secondary Health at a Glance: Europe 2016. Paris: OECD Publishing; 2016. p. 1-193. Edited by Secondary OECD.

36. WHO. World health statistics 2017: monitoring health for the SDGs, Sustainable Development Goals. Geneva: World Health Organization; 2017 p. 1-103.

37. Health-ministry-of-Norway. Regulation of habilitation and rehabilitation, individualized plan and coordinator [Forskrift om habilitering og rehabilitering, individuell plan og koordinator] [https:// lovdata.no/dokument/SF/forskrift/2011-12-16-1256] Accessed on date: 8 Aug 2019

38. Ministry of Health N: White paper 47 (2008-2009). The Coordination Reform. Right treatment, at the right place and time. [Stortingsmelding 47 
(2008-2009), Samhandlingsreformen. Rett behandling - på rett sted - til rett tid.]. In. Edited by Helse-og-Omsorgsdepartementet; 2009: 1-11.

39. Holum LC. The individualized plan as a tool for patient involvement and engagement. An Evaluation report. [Individuell plan som verktøy for brukermedvirkning: En evaluering]. Tidsskrift For Norsk Psykologforening. 2010;47:326-8.

40. Schoen C, Osborn R, Squires D, Doty M, Pierson R, Applebaum S. New 2011 survey of patients with complex care needs in eleven countries finds that care is often poorly coordinated. Health Aff. 2011;30(12):2437-48.

41. Berntsen GR, Høyem A, Lettrem I, Ruland C, Rumpsfeld M, Gammon DB. A person-centered integrated care quality framework. A qualitative study of patients' evaluation of care in light of chronic care ideals. BMC Health Serv Res. 2018;18:1-15.

42. Wagner E, Davis C, Schaefer J, Von Korff M, Austin B. A Survey of Leading Chronic Disease Management Programs: Are They Consistent with the Literature? [Article]. J Nurs Care Qual. 2002;16(2):67-80.

43. Reuben DB, Tinetti ME. Goal-Oriented Patient Care - An Alternative Health Outcomes Paradigm. N Engl J Med. 2012;366:777-9.

44. Andersen ES, Grude KV, Haug T. Goal directed project management. 4th ed. London Philadelphia: Kogan Page; 2009.

45. Elwyn G, Barr PJ, Grande SW, Thompson R, Walsh T, Ozanne EM. Developing CollaboRATE: A fast and frugal patient-reported measure of shared decision making in clinical encounters. Patient Educ Couns. 2013;93:102-7.

46. Vanhaecht K, Panella M, Van Zelm R, Sermeus W. An overview on the history and concept of care pathways as complex interventions. Int J Care Pathways. 2010;14(3):117-23.

47. Lewis S, Smith K. Lessons learned from real world application of the bow-tie method. In: 6th Global Congress on Process Safety: 2010. San Antonio: American Institute of Chemical Engineers; 2010. p. 22-4.

48. PIsek PE, Greenhalgh T. The challenge of complexity in health care. BMJ. 2001;323(7313):625-8.

49. Plsek P. Redesigning health care with insights from the science of complex adaptive systems. In: Crossing the quality chasm: A new health system for the 21st century; 2001. p. 309-22.

50. Snowden DJ, Boone ME. A leader's framework for decision making. Harv Bus Rev. 2007:85(11):1-9.

51. Rosenbaum PR, Rubin DB. Constructing a control group using multivariate matched sampling methods that incorporate the propensity score. Am Stat. 1985;39(1):33-8.

52. Rubin DB. Estimating Causal Effects from Large Data Sets Using Propensity Scores. Ann Intern Med. 1997;127:757-63.

53. Rubin DB. Using propensity scores to help design observational studies: application to the tobacco litigation. Health Serv Outcome Res Methodol. 2001;2(3-4):169-88.

54. Craig P, Dieppe P, Macintyre S, Michie S, Nazareth I, Petticrew M. Medical Research Council G: Developing and evaluating complex interventions: the new Medical Research Council guidance. [see comment]. BMJ. 2008:337:979-83.

55. Greenhalgh T, Papoutsi C. Studying complexity in health services research: desperately seeking an overdue paradigm shift, vol. 16; 2018. p. 1-6.

56. Øvretveit J, Gustafson D. Evaluation of quality improvement programmes. Qual Saf Health Care. 2002;11(3):270-5.

57. CONSORT: Consort 2010 Checklist of information to include when reporting a randomised trial; 2010.

58. von Elm E, Altman DG, Egger M, Pocock SJ, Gotzsche PC, Vandenbroucke JP. The Strengthening the Reporting of Observational Studies in Epidemiology (STROBE) statement: guidelines for reporting observational studies. Lancet. 2007:370(9596):1453-7.

59. Hoffmann TC, Glasziou PP, Boutron I, Milne R, Perera R, Moher D, Altman DG, Barbour V, Macdonald H, Johnston M. Better reporting of interventions: template for intervention description and replication (TIDieR) checklist and guide. BMJ. 2014;348:1-12.

60. Lang TA, Altman DG. Basic statistical reporting for articles published in biomedical journals: the "Statistical Analyses and Methods in the Published Literature" or the "SAMPL Guidelines". Handb Eur Assoc Sci Ed. 2013;256:1-9.

61. Bergmo TS, Berntsen GK, Dalbakk M, Rumpsfeld M. The effectiveness and cost effectiveness of the PAtient-Centred Team (PACT) model: study protocol of a prospective matched control before-and-after study. BMC Geriatr. 2015;15(133):1-9.

62. Brazier J, Harper R, Jones N, O'cathain A, Thomas K, Usherwood T, Westlake L. Validating the SF-36 health survey questionnaire: new outcome measure for primary care. BMJ. 1992;305(6846):160.
63. Turner G, Clegg A. Best practice guidelines for the management of frailty: a British Geriatrics Society, Age UK and Royal College of General Practitioners report. Age Ageing. 2014;43(6):744-7.

64. Linna M. Virtanen M. Chapter 16: NordDRG: The benefits of coordination. In: Busse R, Geissler A, Quentin W, Wiley M, editors. Diagnosis-Related Groups in Europe Moving towards transparency, efficiency and quality in hospitals. Maidenhead, Berkshire: McGrawHill Open University Press; 2011. p. 293-300.

65. Content and data quality in the Norwegian Patient Registry [Innhold og kvalitet i NPR] [https://www.helsedirektoratet.no/tema/statistikk-registre-ograpporter/helsedata-og-helseregistre/norsk-pasientregister-npr/innhold-ogkvalitet-i-npr\#datakvalitet] Accessed on date: 8 Aug 2019

66. Noether GE. Sample size determination for some common nonparametric tests. J Am Stat Assoc. 1987;82(398):645-7.

67. Multiple Linear Regression Analysis, from: Boston University School of public Health [http://sphweb.bumc.bu.edu/otlt/MPH-Modules/BS/ BS704_Multivariable/BS704_Multivariable7.html] Accessed on date: 8 Aug 2018

68. Bias, Confounding and Effect Modification, from Penn State Eberly College of Science [https://newonlinecourses.science.psu.edu/stat507/node/34/] Accessed on date: 8 Aug 2019

69. Rosenbaum PR, Rubin DB. The central role of the propensity score in observational studies for causal effects. Biometrika. 1983;70:41-55.

70. Ho DE. Matchlt: nonparametric preprocessing for parametric causal inference. J Stat Softw. 2011;42(8):1-43.

71. Austin PC. The use of propensity score methods with survival or time-toevent outcomes: reporting measures of effect similar to those used in randomized experiments. Stat Med. 2014;33(7):1242-58.

72. Austin PC. An introduction to propensity score methods for reducing the effects of confounding in observational studies. In: Multivariate Behavioral Research. vol. 46: Taylor \& Francis; 2011. p. 399-424.

73. Billings J, Blunt I, Steventon A, Georghiou T, Lewis G, Bardsley M. Development of a predictive model to identify inpatients at risk of readmission within 30 days of discharge (PARR-30). BMJ Open. 2012;2:1-9.

74. van Walraven C, Austin PC, Jennings A, Quan H, Forster AJ. A modification of the Elixhauser comorbidity measures into a point system for hospital death using administrative data. Med Care. 2009;47(6):626-33.

75. Hilbe J. Modeling count data: Cambridge University Press; 2014. p. 283.

76. Garrett JM. Stcox PH-assumption tests - Tests of proportional-hazards assumption [http://www.stata.com/manuals13/ststcoxph-assumptiontests. pdf] Accessed on date: 16 Aug 2018

77. Higgins JPT, Thompson SG. Quantifying heterogeneity in a meta-analysis. Stat Med. 2002;21:1539-58 John Wiley \& Sons, Ltd.

78. Deeks JJ, Higgins JP, Altman DG. Cochrane Handbook for Systematic Reviews of Interventions. Heterogeneity, chapter 9.5 [https:// handbook-5-1.cochrane.org/chapter_9/9_5_heterogeneity.htm] Accessed on date: 16 Aug 2018

79. Bellika JG, Henriksen TS, Yigzaw KY. The snow system: a decentralized medical data processing system. In: Fernández-Llatas C, García-Gómez $J M$, editors. Data mining in clinical medicine. New York: Springer New York; 2015. p. 109-22.

80. Comprehensive meta analysis [https://www.meta-analysis.com/index. php?cart=BUBR1480837] Accessed on date: 30 Apr 2018

81. Ho DE, Imai K, King G, Stuart EA. Matching as nonparametric preprocessing for reducing model dependence in parametric causal inference. Polit Anal. 2007;15(3):199-236.

82. Armstrong K. Methods in Comparative Effectiveness Research. J Clin Oncol. 2012;30:4208-14.

83. King G, Nielsen R. Why Propensity Scores Should Not Be Used for Matching [https://gking.harvard.edu/files/gking/files/psnot.pdf] Accessed on date: 16 Aug 2018

84. Emanuel EJ, Wendler D, Grady C. What makes clinical research ethical? JAMA. 2000;283(20):2701-11.

85. Sweeney L, Halpert A, Waranoff J. Patient-centered management of complex patients can reduce costs without shortening life. Am J Manag Care. 2007;13(2):84-92.

86. Stanhope V, Tondora J, Davidson L, Choy-Brown M, Marcus SC. Personcentered care planning and service engagement: a study protocol for a randomized controlled trial. Trials. 2015;16:1-11.

87. Gray CS, Wodchis WP, Upshur R, Cott C, McKinstry B, Mercer S, Palen TE, Ramsay T, Thavorn K, Qo CHI. Supporting goal-oriented primary health care 
for seniors with complex care needs using mobile technology: evaluation and implementation of the health system performance research network, bridgepoint electronic patient reported outcome tool. JMIR Res Protoc. 2016;5(2):1-16

88. Dorr DA, Wilcox AB, Brunker CP, Burdon RE, Donnelly SM. The effect of technology-supported, multidisease care management on the mortality and hospitalization of seniors. J Am Geriatr Soc. 2008;56:2195-202.

89. Garåsen H, Windspoll R, Johnsen R. Long-term patients' outcomes after intermediate care at a community hospital for elderly patients. 12month follow-up of a randomized controlled trial. Scand J Public Health. 2008:36:197-204.

90. Saltvedt I, Mo ESO, Fayers P, Kaasa S, Sletvold O. Reduced mortality in treating acutely sick, frail older patients in a geriatric evaluation and management unit. A prospective randomized trial. J Am Geriatr Soc. 2002; 50(5):792-8.

91. Baxter S, Johnson M, Chambers D, Sutton A, Goyder E, Booth A. The effects of integrated care: a systematic review of UK and international evidence. BMC Health Serv Res. 2018;18:2-13.

92. $\mathrm{Xu} X$, Mishra $G D$, Jones $M$. Evidence on multimorbidity from definition to intervention: An overview of systematic reviews. Ageing Res Rev. 2017;37:53-68.

93. Hopman P, de Bruin SR, Joao Forjaz M, Rodriguez-Blazquez C, Tonnara G, Lemmens LC, Onder G, Baan CA, Rijken M. Effectiveness of comprehensive care programs for patients with multiple chronic conditions or frailty: A systematic literature review. Health Policy. 2016;120(7):818-32.

94. Lafont C, Gerard S, Voisin T, Pahor M, Vellas B, Force IAT. Reducing "latrogenic Disability" in the hospitalized frail elderly. J Nutr Health Aging. 2011;15(8):645-60.

95. Blom J, den Elzen W, van Houwelingen AH, Heijmans M, Stijnen T, Van den Hout W, Gussekloo J. Effectiveness and cost-effectiveness of a proactive, goal-oriented, integrated care model in general practice for older people. A cluster randomised controlled trial: Integrated Systematic Care for older People-the ISCOPE study. Age Ageing. 2016;45(1):30-41.

96. Bleijenberg N, Drubbel I, Schuurmans MJ, ten Dam H, Zuithoff NPA, Numans ME, de Wit NJ. Effectiveness of a Proactive Primary Care Program on Preserving Daily Functioning of Older People: A Cluster Randomized Controlled Trial. J Am Geriatr Soc. 2016:64(9):1779-88.

97. Moore GF, Audrey S, Barker M, Bond L, Bonell C, Hardeman W, Moore L, O'Cathain A, Tinati T, Wight D. Process evaluation of complex interventions: medical research council guidance. BMJ. 2015;350:1-7.

\section{Publisher's Note}

Springer Nature remains neutral with regard to jurisdictional claims in published maps and institutional affiliations.

Ready to submit your research? Choose BMC and benefit from:

- fast, convenient online submission

- thorough peer review by experienced researchers in your field

- rapid publication on acceptance

- support for research data, including large and complex data types

- gold Open Access which fosters wider collaboration and increased citations

- maximum visibility for your research: over $100 \mathrm{M}$ website views per year

At BMC, research is always in progress.

Learn more biomedcentral.com/submissions 